\title{
Estudo etnobotânico na comunidade de Conceição-Açu (alto da bacia do rio Aricá Açu, MT, Brasil)
}

\author{
Maria Corette Pasa ${ }^{1,4}$, João Juares Soares ${ }^{2}$ e Germano Guarim Neto ${ }^{3}$
}

Recebido em 19/11/2003. Aceito em 19/08/2004

\begin{abstract}
RESUMO - (Estudo etnobotânico na comunidade de Conceição-Açu (alto da bacia do rio Aricá Açu, MT, Brasil)). O presente trabalho tem como objetivo realizar o levantamento etnobotânico de plantas classificadas em diferentes categorias de uso na comunidade de Conceição-Açu, no município de Cuiabá, MT, Brasil e estimar o valor de uso das espécies botânicas em matas de galeria. Entrevistas estruturadas e semi-estruturadas, o uso do questionário foram aplicados em 59 residentes adultos de ambos os sexos. Identificou-se as unidades de paisagem: quintais, roças e matas de galeria e o número total de espécies utilizadas foi de 180. A maioria das plantas foi coletada durante a entrevista e depositadas no Herbário da UFMT. Nos quintais das residências identificou-se 86 espécies, pertencentes a 43 famílias, a maioria cultivada e utilizada como alimento $(48,1 \%)$ e como remédio $(44,5 \%)$. Nas roças os principais cultivos são: Manihot esculenta L. (100\%), Carica papaya L. (76,2\%), Musa paradisiaca L. (71,4\%), Saccharum officinarum L. (57,1\%). Na mata de galeria destacou-se a categoria medicinal (65\%), com as espécies: Copaifera langsdorffii Desf. (2,5), Aspidosperma polyneuron Muell. Arg. (2,5), Hymenaea stignocarpa Mart. (2,33), Diptychandra aurantiaca Tul. (2,0), Cariniana rubra Gardner ex. Miers (20) e as famílias botânicas Mimosaceae, Bignoniaceae, Caesalpiniaceae, Fabaceae e Sapindaceae. Os resultados demonstram que a população possui vasto conhecimento das plantas e de suas propriedades de cura.
\end{abstract}

Palavras-chave: etnobotânica, categorias, valor de uso, Conceição-Açu

\begin{abstract}
Ethnobotany study in community of Conceição-Açu (on the upper basin of the River Aricá Açu, MT, Brazil)). This paper aims at doing the ethnobotany survey of plants classified in different categories of use in the community of Conceição-Açu, in the city of Cuiabá, MT, Brazil and estimate the value of use of the species botanic in the gallery forest. Structured and non-structured interviews and the questionnaire were used in 59 adult dwellers of both sexes. Were identified the landscape units: backyards, husbandry and gallery forest and the total number of used species is the 180. The majority of the plants was collected during the interview and is kept in the Herbary of the Federal University of Mato Grosso. In the backyards of the house, 86 species, belong 43 families were identified, the majority of which planted and utilized as food (48.1\%) an used as medicinal (44.5\%). In the husbandry the cultivation principal are: Manihot esculenta L. (100\%), Carica papaya L. (76.2\%), Musa paradisiaca L. (71.4\%), Saccharum officinarum L. (57.1\%). In the gallery forest the use value showed the medicinal categoty (65\%) species: Copaifera langsdorffii Desf. (2.5), Aspidosperma polyneuron Muell. Arg. (2.5), Hymenaea stignocarpa Mart. (2.33), Diptychandra aurantiaca Tul. (2.0), Cariniana rubra Gardner ex. Miers (2.0) and the Mimosaceae, Bignoniaceae, Caesalpiniaceae, Fabaceae and Sapindaceae families were the most used. The results show that the populacion has a wide knowledge of the plants and of the his power of healing.
\end{abstract}

Key words: ethnobotany, categories, value of use, Conceição-Açu

\section{Introdução}

A Etnobotânica desponta como o campo interdisciplinar que compreende o estudo e a interpretação do conhecimento, significação cultural, manejo e usos tradicionais dos elementos da flora (Caballero 1979). Segundo Barrera (1979), os estudos etnobotânicos vão além do que pode pretender a investigação botânica, uma vez que suas metas se concentram em torno de um ponto fundamental que é a significação ou o valor cultural das plantas em determinada comunidade humana.

É visível o papel que os povos tradicionais desempenham na exploração dos ambientes naturais, fornecendo informações sobre as diferentes formas de manejo executadas no seu cotidiano e usufruindo da exploração enquanto forma de sustentação desses povos. Assim, diante da marcha da urbanização e das

\footnotetext{
1 Universidade Federal de Mato Grosso, Departamento de Ciências Biológicas, ICEN, CEP 78735-901, Rondonópolis, MT, Brasil

2 Universidade Federal de São Carlos, Departamento de Botânica, Centro de Ciências Biológicas e da Saúde, C. Postal 676, CEP 13565-905, São Carlos, São Paulo, Brasil

3 Universidade Federal de Mato Grosso, Instituto de Biociências, Departartamento de Botânica e Ecologia, Av. Fernando Correa da Costa, s/n, CEP 78060-900, Cuiabá, MT, Brasil

4 Autor para correspondência: pasamc@terra.com.br
} 
possíveis influências da aculturação, é preciso resgatar o conhecimento que a população detém sobre o uso de recursos naturais. Nesse sentido, alguns autores propõem-se estimar o valor de uso das plantas com a finalidade de apontar as espécies e famílias de preferência da população humana no universo vegetal (Phillips \& Gentry 1993; Phillips 1996).

O conhecimento tradicional sobre o uso das plantas é vasto e é, em muitos casos, o único recurso disponível que a população rural de países em desenvolvimento tem ao seu alcance. As plantas usadas como remédio quase sempre têm posição predominante e significativa nos resultados das investigações etnobotânicas de uma região ou grupo étnico.

Este trabalho tem como objetivo catalogar, sistematizar e analisar, de forma integrada, o conhecimento que os moradores rurais da comunidade de Conceição-Açu, MT, Brasil possuem a respeito da flora local, bem como a utilização desses recursos vegetais e a dinâmica que move a interação ser humano-planta.

\section{Material e métodos}

O estudo foi realizado na comunidade rural de Conceição-Açu, situada na baixada cuiabana, no Município de Cuiabá, MT. A área localiza-se a $45 \mathrm{~km}$ ao sul de Cuiabá, no alto da bacia do rio Aricá Açu, afluente do rio Cuiabá (região de APA conforme Decreto Estadual de 27/11/1995). Delimitada pelas coordenadas geográficas $15^{\circ} 30^{\prime}$ e $15^{\circ} 40^{\prime}$ S e $55^{\circ} 35^{\prime}$ e 55 $50^{\prime}$ W pertence à Microregião de Cuiabá e Mesoregião - Centro Sul Mato-grossense (Radambrasil 1982).

Segundo a classificação climática de Köppen, domina na área um sistema de clima pertencente ao Grupo A (Clima Tropical Chuvoso). O tipo climático é dominantemente o Aw, caracterizado por ser quente e úmido, com duas estações definidas: uma estação chuvosa (outubro a março) e outra seca (abril a setembro), que coincide com o inverno. As precipitações médias anuais registram em torno de $2.000 \mathrm{~mm}$ e as temperaturas médias são altas e regulares durante o ano, com médias anuais de $23{ }^{\circ} \mathrm{C}$ a $25^{\circ} \mathrm{C}$. Há um declínio pouco sensível nos meses de julho e agosto. A média das máximas fica entre $30^{\circ} \mathrm{C}$ e $32^{\circ} \mathrm{C}$, sem grandes oscilações durante o ano, embora as mínimas decresçam no inverno e à noite. A formação vegetal predominante é o cerrado, com a presença de floresta decídua na encosta dos morros, com paredões e afloramentos calcáreos representados pela Serra de
Chapada dos Guimarães e mata de galeria ao longo dos rios (PCBAP 1997).

A área de estudo é banhada pelos mananciais hídricos da porção alta e margem esquerda da microbacia do rio Aricá Açu, potencial hídrico da região, caracterizado como um rio de $3^{\mathrm{a}}$ ordem (Strabler \& Strabler 1989). Seus afluentes, caracterizados como de menor porte, são representados pelos rios e córregos denominados Aguaçu, Claro, Glória, Conceição, Tamanduá, Piancó, Barrero, entre outros. O rio Aricá Açu possui extensão de aproximadamente $112 \mathrm{~km}$ e a porção alta da bacia que representa a área de estudo possui $31 \mathrm{~km}$ e área de aproximadamente $413 \mathrm{~km}^{2}$ (Laboratório de Cartografia da UFMT 2003).

A região apresenta solos minerais não hidromórficos, com horizonte $\mathrm{B}$, do vermelho ao amarelo, de textura média a argilosa, de pouco profundo a profundo, de bem a imperfeitamente drenado e o relevo de ondulado, forte ondulado e montanhoso; a unidade típica de paisagem é de savana com mata de galeria (PCBAP 1997).

As principais atividades econômicas são a agricultura de subsistência, a fabricação de farinha de mandioca, a pesca e a caça para o consumo familiar.

A região apresenta certo isolamento em relação à área urbana, o que não impede a penetração das concepções do mundo atual influenciadas pelo meio social circundante, sob a força da urbanização.

Os dados foram coletados de outubro/2001 a 17/dezembro/2002, com freqüência de visitas semanais, inicialmente, e quinzenais nos últimos seis meses. Utilizou-se questionário, observação participante, entrevistas semi-estruturadas e estruturadas (Minayo 1994) para obtenção de características sócioeconômicas dos informantes, características botânicas e ecológicas das plantas usadas para fins medicinais e suas indicações terapêuticas. Foram entrevistados 59 pessoas, 27 mulheres, 27 homens e cinco jovens: quatro do gênero masculino e um do gênero feminino, na faixa etária entre 20 e 25 anos de idade. A maioria dos informantes possui idade acima de 55 anos. $O$ grau de escolaridade é baixo, a maioria cursou no máximo até a $4^{a}$ série do ensino fundamental e $30 \%$ da amostra não possui escolaridade alguma. Os informantes demonstraram interesse em participar da pesquisa porque usam as plantas para alguma finalidade.

Durante a coleta dos recursos vegetais, do registro fotográfico, das gravações em fita cassete e das anotações durante as expedições detectou-se as seguintes unidades de paisagem utilizadas pela comunidade local: roças, hortas e quintais, e mata de 
galeria. As hortas localizam-se dentro dos quintais, próximo da casa, facilitando seu uso. As roças contornam as matas de galeria.

As exsicatas do material botânico foram identificadas pelos especialistas do Departamento de Botânica e Ecologia do Instituto de Biociêncas, Prof. Dr. Germano Guarim Neto e do Herbário Central da Universidade Federal de Mato Grosso (UFMT) pela Profa. Dra. Miramy Macedo, onde encontram-se catalogadas e depositadas.

Conforme as entrevistas, os informantes mostravam os objetos e as plantas usadas, as quais foram classificadas em diferentes categorias de uso pela comunidade local, ou seja, como alimento, remédio, ornamental, proteção, fabricação de utensílios domésticos (bancos, cadeiras, mesas, cama, prateleiras, nicho religioso) e também de utensílios de trabalho (cabo de enxada, de foice e de facão).

As espécies com aplicação terapêutica consideradas neste trabalho incluem, além daquelas indicadas para as doenças que têm correspondência com a medicina oficial, outras, como, por exemplo, olho-gordo, inveja, quebrante, uma vez que fazem parte do universo nosológico da comunidade estudada, porém, não reconhecidas pela biomedicina. As indicações foram agrupadas com base na classificação das doenças proposta pela Organização Mundial de Saúde (OMS 2000).

Para se calcular o valor de uso empregou-se:

a. o valor de uso de cada espécie ( $s p$ ) por informante (i), dado como:

$$
\mathrm{VU} s p i=\frac{\sum \mathrm{U} s p i \mathrm{~N} s p i}{\mathrm{~N} s p i}
$$

onde, Uspi é o número de usos mencionados por informante $i$ por espécie $s p$ em cada evento, e Nspi é o número de eventos com o informante $i$ por espécie $s p$.

b. o valor de uso global de cada espécie (VUsp), dado como:

$$
\mathrm{VU} s p=\frac{\sum \mathrm{VU} s p i}{\mathrm{n} s}
$$

onde, $N s$ é o numero de informantes entrevistados por espécie.

\section{Resultados e discussão}

As roças - As roças representam as áreas de produção agrícola cuja formação ocorre nas áreas abertas no interior da vegetação natural, principalmente com a derrubada da mata ripária ou nas bordas do campo cerrado fazendo limite com as matas.
A comunidade é composta por 24 famílias de moradores locais, com população de aproximadamente 90 pessoas, caracterizadas, na sua maioria, por situação sócio-econômica baixa, ou seja, sitiantes que se ligam aos cultivos de lavoura em escala reduzida, apenas para subsistência.

As áreas de manejo agrícola são representadas pelas unidades familiares. As atividades agrícolas praticadas pelos moradores rurais são, principalmente, as culturas de cana-de-açúcar, mandioca, banana, mamão, melancia, milho e feijão. A agricultura de subsistência, nessa região, é considerada atividade fundamental, sendo diversificada na maioria das unidades de produção familiar (55\%). Entre os entrevistados, o número mínimo de explorações agrícolas é de três (mandioca, mamão e banana) e o máximo sete (feijão, milho, mandioca, banana, mamão, melancia e cana-de-açúcar). Apenas $14,2 \%$ dos entrevistados dispõem-se ainda a plantar o milho e o feijão e 9,5\% plantam o arroz, mas não em todos os anos.

Entre os produtos cultivados, a mandioca constitui o cultivo principal (Tab. 1) e caracteriza-se como exploração tipicamente regional, sem fins de comercialização. Dados semelhantes foram reportados em Santo Antônio do Leverger (MT) quanto ao cultivo da mandioca (Amorozo 2000) e entre os caiçaras da Mata Atlântica (Begossi 2001). O processamento doméstico da mandioca para a produção de farinha é de pequena escala e artesanal, apenas para o consumo familiar, sendo o método tradicional que vigora.

A área de plantio diversificado nas roças de Conceição-Açu varia de 0,4 ha até 2 ha por unidade familiar. As atividades referentes à roça não apresentam grandes distinções na divisão sexual do

Tabela 1. Cultivos existentes em roças na comunidade de Conceição-Açu, Cuiabá, MT, Brasil.

\begin{tabular}{clc}
\hline Propriedades $\left(\mathrm{n}^{\circ}\right)$ & Cultivos & Freqüência (\%) \\
\hline 21 & mandioca & 100,0 \\
16 & mamão & 76,2 \\
15 & banana & 71,4 \\
15 & laranja & 71,4 \\
15 & limão & 71,4 \\
12 & cana-de-açúcar & 57,1 \\
11 & melancia & 52,3 \\
09 & batata doce & 42,8 \\
03 & milho & 14,2 \\
03 & abacaxi & 14,2 \\
03 & feijão & 14,2 \\
02 & arroz & 9,5 \\
\hline
\end{tabular}


trabalho, sendo que $10 \%$ do total das propriedades na região são administradas somente por mulheres. Os cuidados dispensados à manutenção das lavouras envolvem a limpeza constante da área de plantio, a qual é executada com frequiência diária, que varia de uma a duas vezes. Ainda em relação aos cuidados dispensados às roças é importante registrar a presença da fauna nativa na época da seca. Este comportamento constitui-se um padrão sazonal resultante da ação conjunta de fatores climáticos e ecológicos (época de seca) que se traduz pela diminuição ou mesmo falta de alimentos nas matas de galeria da região, representando uma soma de habitats de borda, quado ocorre a invasão dos animais nas roças.

Para manter o estoque genético das espécies plantadas nas roças, as sementes são obtidas, geralmente, na propriedade. Igualmente as mudas de mandioca e outras espécies são oriundas da propriedade. A troca, entre parentes e vizinhos, constitui prática bastante comum na região, não sendo comum a compra de sementes ou mudas de plantas.

As pessoas de saber local são simples e trabalham sem tecnologia (65\%), não possuem equipamentos modernos para os trabalhos na agricultura de subsistência. Não possuem técnicas aprimoradas e encontram-se limitadas pela escala de produção imposta pelo tamanho da propriedade e pela falta de infra-estrutura e mão-de-obra; sendo assim, são obrigadas a manter a produção diversificada, que conta com a força da mão-de-obra familiar como uma atividade locus de produção e sobrevivência da família. É comum entre os moradores de Conceição-Açu o uso dos recursos naturais como insumos. $\mathrm{O}$ esterco de animais domésticos é usado como adubo entre os moradores num índice de 78\%. Restos de alimentos são usados por $55 \%$ da população. Entre as técnicas utilizadas, os moradores locais que fazem rotação de cultura representam $70 \%$ da população e $55 \%$ dos moradores locais fazem o plantio consorciado. Em termos de conservação dos recursos a prática do pousio constitui estratégia que visa a ressignificação da fertilidade da terra em se tratando das condições da mesma para novos plantios.

Os quintais - Outro espaço que colabora para a subsistência da família é o quintal, que exerce considerável papel econômico na vida das pessoas. Em geral, os quintais de Conceição-Açu localizam-se atrás da residência, sendo de tamanho suficiente para atender a demanda familiar; são constituídos por grande número de espécies perenes e riqueza de espécies que permite a produção ao longo do ano. Além disso, os quintais possuem cobertura vegetal diversificada sobre o solo. A produção vegetal dos quintais e hortas na região pode ser dividida em espécies (1) frutíferas, (2) hortaliças, (3) medicinais. Todas as casas possuem um quintal que serve, entre outras funções, como fonte de alimento e de remédio. É por meio da produção hortifrutífera dos quintais que a população mantém a baixa dependência de produtos adquiridos externamente, porque os quintais são aptos para fornecer produtos para uso local, bem como contribuir para a economia regional por meio dos produtos que este espaço oferece; ocasiona impactos mínimos sobre o ambiente, ao desempenhar várias funções ecológicas, incluindo benefícios hidrológicos, modificações microclimáticas e controle da erosão do solo, além da conservação dos recursos genéticos; conserva os recursos vegetais e a diversidade cultural, fundamentada no saber e na cultura dos moradores locais, recebendo tratamento especial ao ser utilizado como espaço para atividades variadas como: de trabalho, de encontros, de festas, rezas e cerimônias; utiliza os insumos naturais como os dejetos animais, restos de vegetais, como folhas e raízes, cinza, terra transportada da mata de galeria promovendo o fortalecimento do espaço ocupado com a variedade de plantas.

A riqueza vegetal encontrada nos quintais das 21 residências de Conceição-Açu foi de 83 espécies, representadas por 43 famílias botânicas (Tab. 2), a maioria registrada praticamente em todos os quintais. As etnocategorias citadas refletem a demanda das necessidades básicas de cada família, ou seja, alimentação, medicinal, ornamental, lenha, fibra, utensílios e cerimoniais, refletindo a influência cultural dos moradores na seleção de espécies introduzidas nos quintais.

Das espécies usadas nos quintais, as frutíferas $(48,1 \%)$ destacam-se na categoria alimentar, estando presentes em todos os quintais. As mais freqüentes são a manga, o caju, a goiaba, a laranja e o limão. Essas frutas fornecem boa parte das necessidades calóricas à dieta dos moradores locais. Dados semelhantes foram registrados nas comunidades de Varginha, Barreirinho e Morro Grande (MT) por Amorozo (2000).

$\mathrm{Na}$ categoria de uso ornamental, obteve-se $25 \%$ do total das espécies usadas. Mais da metade das espécies utilizadas como alimento são também consideradas ornamentais, como é o caso da mangueira, jaboticabeira, cajueiro e citros, destacando-se como importantes elementos estruturais desse sistema. 
Tabela 2. Espécies existentes nos quintais de Conceição-Açu, Cuiabá, MT, Brasil. Usos: Al = Alimento; Re = Remédio; Or = Ornamental; Ot $=$ Outros .

\begin{tabular}{|c|c|c|c|}
\hline Nome científico & Nome vulgar & Família & Usos \\
\hline Persea americana Mill. & abacate & Lauraceae & $\mathrm{Al}$ \\
\hline Ananas comosus (L.) Merril & abacaxi & Bromeliaceae & $\mathrm{Al}$ \\
\hline Cucurbita pepo L. & abóbora & Cucurbitaceae & $\mathrm{Re} ; \mathrm{Al}$ \\
\hline Malpighia glabra L. & acerola & Malpighiaceae & $\mathrm{Al}$ \\
\hline Lactuca sativa $\mathrm{L}$ & alface & Asteraceae & $\mathrm{Al}$ \\
\hline Gossypium herbaceum L. & algodão & Malvaceae & $\operatorname{Re}$ \\
\hline Cichorium intybus $\mathrm{L}$. & almeirão & Asteraceae & $\mathrm{Al}$ \\
\hline Morus nigra $\mathrm{L}$. & amora & Moraceae & $\mathrm{Al}$ \\
\hline Artemisia absinthium $\mathrm{L}$. & atermije, losna & Asteraceae & $\operatorname{Re}$ \\
\hline Ruta graveolens $\mathrm{L}$. & arruda & Rutaceae & $\mathrm{Re} ; \mathrm{Ot}$ \\
\hline Vernonia ferruginea Less. & assa-peixe & Asteraceae & $\operatorname{Re}$ \\
\hline Adiantum sp. & avenca & Adiantaceae & Or \\
\hline Alloe vera $\mathrm{L}$ & babosa & Liliaceae & $\operatorname{Re}$ \\
\hline Musa parasidiaca Kuntze & banana & Musaceae & $\mathrm{Al}$ \\
\hline Ipomoea batatas L. Lam. & batata-doce & Convolvulaceae & $\mathrm{Al}$ \\
\hline Begonia sp. & begônia & Begoniaceae & Or \\
\hline Coleus barbatus (Andrews) Benth. & boldo & Lamiaceae & $\operatorname{Re}$ \\
\hline Brachiaria sp. & braquiária & Poaceae & Or \\
\hline Cactus sp. & cacto & Cactaceae & Or \\
\hline Coffea arabica $\mathrm{L}$. & café & Rubiaceae & $\mathrm{Al}$ \\
\hline Vernonia polianthes $\mathrm{L}$. & caferana & Asteraceae & $\operatorname{Re}$ \\
\hline Spondias dulcis $\mathrm{L}$. & cajá-manga & Anacardiaceae & $\mathrm{Re} ; \mathrm{Al}$ \\
\hline Anacardium occidentale $\mathrm{L}$. & caju & Anacardiaceae & $\mathrm{Al}$ \\
\hline Matricaria recutita $\mathrm{L}$. & camomila & Asteraceae & $\operatorname{Re}$ \\
\hline Saccharum officinarum $\mathrm{L}$. & cana-de-açúcar & Poaceae & $\mathrm{Al}$ \\
\hline Bambusa vulgaris L. & cana-taquara, taboca & Poaceae & $\mathrm{Al}$ \\
\hline Cymbopogum citratus L. & capim-cidreira & Poaceae & $\mathrm{Re}$; Or \\
\hline Jacaranda semiserrata $\mathrm{C}$. & carobinha-do-campo & Bignoniaceae & $\operatorname{Re}$ \\
\hline Baccharis trimera (Less.) DC. & carqueja & Asteraceae & $\operatorname{Re}$ \\
\hline Allium choenoprasum $\mathrm{L}$. & cebolinha & Liliaceae & $\mathrm{Al}$ \\
\hline Daucus carota L. & cenoura & Apiaceae & $\mathrm{Al}$ \\
\hline Cichorium endivia $\mathrm{L}$. & coentro & Asteraceae & $\mathrm{Al}$ \\
\hline Cocos nucifera $\mathrm{L}$. & coco-da-bahia & Arecaceae & $\mathrm{Al} ; \mathrm{Or}$ \\
\hline Alpinia speciosa Schum. & colônia & Zingiberaceae & $\operatorname{Re}$ \\
\hline Dieffenbachia picta Schott. & comigo-ninguém-pode & Araceae & Or; Ot \\
\hline Symphytum officinale L. & confrei & Boraginaceae & $\operatorname{Re}$ \\
\hline Brassica oleracea DC. & couve & Brassicaceae & $\mathrm{Al}$ \\
\hline Lippia alba (Mill.) Blume & erva-cidreira & Verbenaceae & $\operatorname{Re}$ \\
\hline Polygonum acre H.B.K. & erva-de-bicho & Polygonaceae & $\operatorname{Re}$ \\
\hline Sansevieria trifasciata Hort. & espada-de-são-jorge & Liliaceae & Or; Ot \\
\hline Spathodea campanulata $\mathrm{L}$. & espatódia & Bignoniaceae & Or \\
\hline Spinacea oleracea $\mathrm{L}$. & espinafre & Chenopodiaceae & $\mathrm{Al}$ \\
\hline Eucalyptus spp. & eucalipto & Myrtaceae & $\mathrm{Re}$; Or \\
\hline Cassia occidentale L. & fedegoso & Caesalpiniaceae & $\operatorname{Re}$ \\
\hline Zingiber officinalis Rosc. & gengibre & Zingiberaceae & $\operatorname{Re}$ \\
\hline Sesamum indicum DC. & gergelin & Pedaliaceae & $\mathrm{Al}$ \\
\hline Psidium guajava $\mathrm{L}$. & goiaba & Myrtaceae & $\mathrm{Al}$; Or \\
\hline Petiveria alliacea $\mathrm{L}$. & guiné & Phytolaccaceae & $\mathrm{Re} ; \mathrm{Ot}$ \\
\hline Mentha piperita $\mathrm{L}$. & hortelã & Lamiaceae & $\operatorname{Re}$ \\
\hline Myrciaria cauliflora (Mart.) O. Berg. & jaboticaba & Myrtaceae & $\mathrm{Al}$ \\
\hline Arthocarpus integrifolia $\mathrm{L}$. & jaca & Moraceae & $\mathrm{Al}$ \\
\hline Genipa americana $\mathrm{L}$. & jenipapo & Rubiaceae & $\mathrm{Al}$ \\
\hline Stachytarpheta augustifolia Lopez-Palacios & jervão & Verbenaceae & $\operatorname{Re}$ \\
\hline Solanum aff. lycocarpum A. St.-Hil. & jurubeba & Solanaceae & $\operatorname{Re}$ \\
\hline Citrus aurantium L. & laranja & Rutaceae & $\mathrm{Al}$; Or \\
\hline Citrus limetta $\mathrm{R}$. & laranja-lima & Rutaceae & $\mathrm{Al}$; Or \\
\hline
\end{tabular}


Tabela 2 (continuação)

\begin{tabular}{|c|c|c|c|}
\hline Nome científico & Nome vulgar & Família & Usos \\
\hline Citrus limonum Osbeck & limão & Rutaceae & $\mathrm{Re} ; \mathrm{Al} ; \mathrm{Or}$ \\
\hline Carica papaya $\mathrm{L}$. & mamão & Caricaceae & $\mathrm{Al}$ \\
\hline Manihot esculenta $\mathrm{L}$. & mandioca & Euphorbiaceae & $\mathrm{Al}$ \\
\hline Mangifera indica $\mathrm{L}$. & manga & Anacardiaceae & $\mathrm{Al} ; \mathrm{Or}$ \\
\hline Passiflora edulis Sims. & maracujá & Passifloraceae & Re; Al; Or \\
\hline Achyrocline satureoides (Lam.) DC. & macela & Asteraceae & $\operatorname{Re}$ \\
\hline Chenopodium ambrosioides $\mathrm{L}$. & mastruz & Chenopodiaceae & $\mathrm{Re}$ \\
\hline Cucumis melo $\mathrm{L}$. & melão & Cucurbitaceae & $\mathrm{Al}$ \\
\hline Zea mays L. & milho & Poaceae & $\mathrm{Al}$ \\
\hline Vanilla planifolia Andr. & orquídea & Orchidaceae & Or \\
\hline Cucumis sativus L. & pepino & Cucurbitaceae & $\mathrm{Al}$ \\
\hline Mentha pulegium $\mathrm{L}$. & poejo & Lamiaceae & $\mathrm{Re}$ \\
\hline Phyllanthus orbiculatus Rich. & quebra-pedra & Euphorbiaceae & $\mathrm{Re}$ \\
\hline Punica granatum $\mathrm{L}$. & romã & Punicaceae & $\mathrm{Re} ; \mathrm{Or}$ \\
\hline Eruca sativa Mill. & rúcula & Brassicaceae & $\mathrm{Al}$ \\
\hline Sambucus nigra L. & sabugueiro & Caprifoliaceae & $\operatorname{Re}$ \\
\hline Petroselinum crispum (Mill.) Nyman & salsa & Apiaceae & $\mathrm{Al}$ \\
\hline Smilax sp. & salsaparrilha & Smilacaceae & $\mathrm{Re}$ \\
\hline Polypodium sp. & samambaia & Polypodiaceae & Or \\
\hline Terminalia cattapa $\mathrm{L}$. & sete-copas & Combretaceae & Or \\
\hline Colocasia esculenta L. & taioba & Araceae & $\mathrm{Re}$ \\
\hline Tamarindus indica $\mathrm{L}$ & tamarindo & Caesalpiniaceae & $\mathrm{Al}$ \\
\hline Lycopersicum esculentum Mill. & tomate & Solanaceae & $\mathrm{Re} ; \mathrm{Al}$ \\
\hline Bactris glaucescens Drude & tucum & Arecaceae & $\operatorname{Re}$ \\
\hline Bixa orellana $\mathrm{L}$ & urucum & Bixaceae & $\mathrm{Al}$ \\
\hline Cereus peruvianus Mill. & urumbeba & Cactaceae & Or \\
\hline Scoparia dulcis L. & vassourinha & Scrophulariaceae & $\operatorname{Re}$ \\
\hline
\end{tabular}

As famílias Rutaceae, Myrtaceae, Cactaceae e Poaceae foram as mais frequientes nessa categoria.

Plantas usadas como remédio representam $44,5 \%$ do total das espécies existentes nos quintais da região. As famílias mais freqüentes foram Asteraceae, Lamiaceae, Liliaceae, Solanaceae e Verbenaceae. As espécies medicinais mais freqüentes nos quintais da região foram o boldo, a carqueja, a atermije, o mastruz, o poejo, o quebra-pedra, o confrei, a camomila, o capimcidreira, entre outros. Resultados semelhantes foram encontrados por Guarim-Neto $(1984 ; 1996)$ e Berg (1980). Algumas destas espécies, na sua maioria herbáceas, possivelmente foram introduzidas por imigrantes, devido à sua facilidade de transporte de uma região para outra.

Arruda, comigo-ninguém-pode, espada-de-são jorge e guiné foram as espécies classificadas como de proteção, baseado no uso em crenças e ritos espirituais, representando as espécies ditas dos "cerimoniais".

Do total de 83 espécies vegetais existentes nos quintais das residências de Conceição-Açu, 37 espécies foram indicadas como remédio no uso terapêutico que abrange as diferentes categorias referentes às afecções do organismo. Para estas espécies vegetais foram atribuídas 29 funções terapêuticas para os diferentes sistemas do corpo humano, entre estas: vermes, inflamação do útero, inflamação do ovário, febre, dor de cabeça, abortivo, diurético, úlcera, gastrite, tontura, derrame, pressão alta, vômito, diarréia, tosse, gripe, hemorróida, azia, varizes, machucadura, quebradura, etc. (Tab. 3). A parte da planta usada como remédio que obteve o maior percentual foi a folha (69\%), seguida da flor $(5,5 \%)$, raiz $(5,5 \%)$, sumo, casca do caule, fruto, semente e bulbo, 2,8\% cada uma. Entre as formas de preparo, a mais expressiva é o chá, com $81 \%$; outras formas também foram relatadas como infusão, compressas, banhos, macerado, emplastro e ao natural.

Entre as plantas usadas como remédio verificou-se que a maioria destina-se ao tratamento de problemas referentes ao aparelho digestivo (25\%), como auxiliares de digestão, prisão de ventre, diarréia, estômago e fígado, seguido pelas afecções ocasionadas por infecção e parasitas $(16,6 \%)$, aparelhos circulatório e geniturinário $(16,2 \%$ cada), sistema tegumentar $(8,3 \%)$ e sistema nervoso e aparelho respiratório, com 5,5\% cada. Outras afecções, como do sistema osteomuscular e tecido conjuntivo, afecções do sangue e órgão 
Tabela 3. Espécies botânicas cultivadas nos quintais usadas como remédio e as afecções orgânicas referidas. Conceição-Açu, Cuiabá, MT, Brasil.

\begin{tabular}{lll}
\hline Categoras & Espécies & Usos \\
\hline $\begin{array}{l}\text { Doenças do sistema nervoso } \\
\text { Doenças do aparelho digestivo }\end{array}$ & $\begin{array}{l}\text { capim-cidreira, maracujá } \\
\text { boldo, caferana, losna, carqueja, } \\
\text { limão, hortelã, jurubeba, camomila, } \\
\text { macela }\end{array}$ & $\begin{array}{l}\text { Ansiedade, agitação } \\
\text { Digestão, dor de estômago, dor de barriga, } \\
\text { azia, mal-estar, fígado, ânsia de vômito }\end{array}$ \\
$\begin{array}{ll}\text { Doenças do aparelho respiratório } \\
\text { Doenças do aparelho circulatório }\end{array}$ & $\begin{array}{l}\text { colônia, limão, erva-de- bicho, confrei } \\
\text { Doenças do aparelho genitourinário }\end{array}$ & $\begin{array}{l}\text { Pressão alta, sangue grosso, hemorróida, } \\
\text { limpar o sangue }\end{array}$ \\
$\begin{array}{ll}\text { tomate, algodão, quebra-pedra, romã, } \\
\text { vassourinha, carobinha-do-campo }\end{array}$ & $\begin{array}{l}\text { Diurético, inflamação dos ovários, útero } \\
\text { bexiga, urina, menstruação, menopausa } \\
\text { Doenças infecciosas e parasitárias }\end{array}$ & $\begin{array}{l}\text { Coceiras, alergia, manchas } \\
\text { arruda, guiné, taioba }\end{array}$ \\
$\begin{array}{ll}\text { abóbora, hortelã, mastruz, jervão, } \\
\text { camomila, cajá-manga } \\
\text { tucum }\end{array}$ & $\begin{array}{l}\text { Vermão intestinal } \\
\text { conjuntivo }\end{array}$ & Energético \\
$\begin{array}{l}\text { Transtornos mentais e comportamentais } \\
\text { Doenças do sangue e órgãos hematopoiéticos }\end{array}$ & $\begin{array}{l}\text { erva- cidreira } \\
\text { salsaparrilha }\end{array}$ & $\begin{array}{l}\text { Calmante } \\
\text { Limpa o sangue }\end{array}$ \\
\hline
\end{tabular}

hematopoiéticos, transtornos mentais e comportamentais representam, cada uma delas, $2,7 \%$ das utilizações obtidas. Essas referências aos tratamentos de saúde local são confirmadas pelos diagnósticos emitidos pela Coordenadoria de Saúde Rural, segundo dados do Relatório Anual. A mesma atende à saúde por meio da prestação de serviços ambulatoriais da Fundação da Saúde de Cuiabá - Coordenadoria de Saúde Rural da Prefeitura Municipal de Cuiabá.

A mata de galeria - A presença da mata de galeria, particularmente no cerrado mato-grossense, reveste-se de grande importância na vida da população regional. De um lado, pela oferta de remédios e alimentos para a subsistência das famílias; de outro, por ser um dos vetores que leva determinados moradores locais à conservação dos recursos nela existentes e, com ela identificam-se socialmente, enquanto membro da comunidade.

A mata de galeria é considerada pela população local um componente essencial e fundamental à unidade paisagística que caracteriza a região. Nela encontram-se presentes os representantes da flora e da fauna local que são considerados símbolos vivos não só de uma geração, mas de várias gerações.

Na região estudada, as matas de galeria estendem-se por toda a extensão no alto da microbacia do rio Aricá Açu e são drenadas pelos rios que nascem na serra da Chapada dos Guimarães e correm em direção ao rio Aricá Açu. Tanto as matas de galeria quanto os rios presentes em cada propriedade lhes confere o valor patrimonial dos recursos naturais nela existente, os quais são expressos pelas seguintes afirmações:

“... das matas nós tira de tudo, a madeira, a comida, os remédios, tem comida pra nós e pros bichos também... no tempo da seca é mais difícil os bichos saem prá buscá comida... no tempo das chuva tem mais fartura... na mata sempre tem água pros bichos ..." (Sra. D.A.A. da S., 69 anos, comunidade de Conceição-Açu, Cuiabá, MT, 2002).

“... Se acabá com a mata, acaba a sombra, acaba a água, a comida, acaba a fartura de tudo que se busca lá (na mata)... o causo é que a gente e os bichos depende de tudo que tem na mata... é uma riqueza muito grande que a gente tem na vida, a gente tem que dá muito valor pra ela (mata) tem que zelá”. (Sr. D.S.R. da S., 64 anos, comunidade de Conceição-Açu, Cuiabá, MT).

Quanto à coleta de produtos na mata de galeria, presenciou-se o processo de extração do óleo de copaíba, o qual é retirado do cerne da árvore (Copaifera langsdorffii), muito utilizado com finalidades medicinais. Para Almeida \& Albuquerque (2002) esta espécie obteve um dos mais altos índices quanto ao valor de importância relativa (IR) das plantas conhecidas como medicinais na Feira de Caruaru, Agreste de Pernambuco. Em Conceição-Açu, a atividade de coleta acontece por meio de mutirão. Para 
execução da coleta são observados itens considerados fundamentais como a idade da árvore, a altura, o diâmetro do tronco, localização da área a que pertencem as plantas selecionadas e a localização espacial da espécie na comunidade vegetal. Após identificadas e marcadas as árvores escolhidas, a próxima etapa é o processo de extração.

Durante o processo de extração os participantes não olham para cima, não falam alto, não gritam, não riem ou fazem qualquer outro tipo de manifestação efusiva “... não pode pertubá o ambiente... ela (árvore) esconde o líquido da gente” (Sr. P.P., 66 anos. Comunidade de Conceição Açu. Cuiabá, MT, 2002). No entendimento desses moradores locais, essas atitudes estariam perturbando a energia que conserva a natureza em equilíbrio, estariam desrespeitando a "força maior" das matas e assim promovendo a desconcentração espiritual e mental dos extratores, a diluição do óleo e a diminuição da sua quantidade. "Não pode estar ventando muito porque o vento promove o "espalhamento" do óleo da árvore para os galhos $e$ as folhas e com isso provoca a redução na quantidade do produto a ser coletado". O material usado para a extração do óleo compõe-se do trado, mangueira de pequeno calibre, balde, frascos ou recipientes de vidro e cortiça. Conversam em voz baixa para definir a área e a altura da perfuração no tronco da árvore. Dá-se o início à perfuração do tronco através do manivelar de um trado que o fura até o cerne. $\mathrm{O}$ tempo dispensado à etapa da coleta de óleo pode variar de árvore para árvore, conforme a quantidade de óleo existente, e demorar de duas até cinco ou seis horas.

O óleo é extraído, geralmente, de uma ou duas árvores apenas e a extração é sazonal, preferencialmente no mês de agosto de cada ano. Assim acontece porque esse período representa o final da época seca, significando que o óleo encontra-se mais concentrado, mais consistente. Apresenta aspecto viscoso, coloração amarelada e aroma agradável. O óleo é usado como antinflamatório, para gripes, tosses, resfriados, dores no corpo, para tratar de feridas abertas, quebraduras e alergias.

Outras espécies presentes nas matas da região, como Calophyllum brasiliensis, Hymenea stignocarpa, Tabebuia heptaphylla, Bixa orellana, Protium heptaphyllum, Croton salutaris, Cariniana rubra, Inga sp., são bastante conhecidas e utilizadas pela população local. São árvores de terra firme e de certos lugares úmidos e arenosos. Sua madeira é usada para confecção de móveis, prateleiras, bancos, cercas e moirões. Alguns frutos são comestíveis e usados como remédio na medicina caseira. Um estudo que aborda o uso dos recursos vegetais da caatinga, no agreste de Pernambuco, revela que entre os usuários das plantas locais o jatobá é altamente utilizado na região de Alagoinha (PE), demonstrando a importância da espécie como medicinal para a comunidade local (Albuquerque \& Andrade 2002).

Na região de Conceição-Açu o trabalho nas matas de galeria encerra dimensões múltiplas, reunindo elementos que fazem parte da percepção que as pessoas detêm sobre as plantas do seu cotidiano, associado aos elementos atrelados ao mundo mágico, ritual, espiritual, enfim, simbólico. A natureza é um lugar de permanente observação e reprodução de saberes. A mata de galeria é percebida como unidade fundamental na paisagem da região desenhada por seus contornos hídricos e florísticos que lhe atribuem valor substancial ao cenário ribeirinho e rural de ConceiçãoAçu. As espécies vegetais que compõem a flora são descritas pela função que exercem, pela disponibilidade de acesso, pela cor, pela forma, pelo aroma, pelo sabor, pelos frutos e pelas folhas.

A bagagem classificatória encontrada pelas pessoas que vivem próximo das matas de galeria em Conceição-Açu permite descrever as plantas do ser humano, as plantas de animais de caça, as plantas de peixes segundo suas características percebidas pelo conhecimento visual, tátil e olfativo adquirido no seu cotidiano. Alguns memes de pessoas entrevistadas referem o conhecimento empírico de objetos da natureza.

“... Os frutos do jatobá é bom pra peixe... O que escorre do tronco do jatobá parece sangue puro... Quando colhe no vidro é igual a sangue de gente... não tem deferença...por isso a seiva dele(do jatobá) é bom pra curá anemia nas pessoas... o sangue do jatobá e das pessoas se parecem por demais... " (Sra. A.M.P., 82 anos, comunidade de Conceição-Açu, Cuiabá, MT, 2002).

“... toda planta tem seu uso pra pessoa, no causo e até pros bichos, tem a folha, tem a raiz, tem o tronco. A gente conhece cada uma olhando bem pras folha, amassa as folhas e já sente o cheiro, se a casca tem cheiro ardido... a gente até exprementa na língua para sabê o tipo de cada planta, assim não erra... o óleo da copaíba é bom pra pessoa e pras machucaduras nos bichos, serve pra tudo..." (Sr. M.A.M., 78 anos, comunidade de Conceição Açu, Cuiabá, MT, 2002).

Em Conceição-Açu as plantas são reconhecidas pelos nomes vulgares (Tab. 4) que definem as 
diferentes espécies ou mesmo as variedades de uma mesma espécie. Assim, as pessoas identificam a planta em função da utilização das partes da mesma para determinado fim. Além disso, investem o seu conhecimento em função da quantidade de indivíduos da espécie como forma de conservar os vegetais enquanto recurso de uso para sua subsistência. Transcreve-se a referência de alguns entrevistados:

“... o óleo da sangra d'água serve para remédio, ele cura as ferida... fecha as feridas depressa... mais não pode usá logo cedo da machucadura, tem de esperá uns dias e aí já pode bebê ou passa dele na ferida...essa árvore tem bastante na beira do rio" (Sra. D.G.D., 53 anos, comunidade de Conceição-Açu, Cuiabá, MT, 2002).

“... a casca do catipé é boa pra fazer o sabão preto... sabão muito bom pra lavá... lava até as ferida, as machucadura... limpa tudo..." (D.A.A. da S., 59 anos, comunidade de Conceição-Açu, Cuiabá, MT, 2002).

O valor dispensado a cada planta varia de acordo com a multiplicidade de sua utilização. Existem plantas que têm diferentes partes utilizadas, como a raiz, o tronco e as folhas. Outras apenas as raízes ou as folhas. Uma espécie vegetal pode pertencer a várias categorias, podendo, por exemplo, apresentar usos alimentar, medicinal e ornamental. O seu valor de uso será, então, diretamente proporcional ao número de usos. As pessoas locais referiram diferentes categorias básicas de uso, tais como: alimento, remédio, ornamental, proteção, madeireira (construção de utensílios de trabalho e domésticos), tecnologia (móveis, sabão) e rituais religiosos ou cerimoniais (Tab. 4).

Na dinâmica de classificação das plantas por valoração utilitária entre os moradores de ConceiçãoAçu o número de famílias botânicas citadas foi de 43 . Na categoria alimento, 11 famílias botânicas $(25,6 \%)$ destacando-se Arecaceae e Rubiaceae (três espécies cada), o restante das famílias representadas com uma espécie cada. Na categoria remédio $(65,6 \%)$, destacando-se as famílias Mimosaceae (oito espécies), Bignoniaceae (sete espécies), Caesalpiniaceae (seis espécies), Fabaceae e Sapindaceae (quatro espécies cada), Apocynaceae, Araceae, Asteraceae, Dilleniaceae, Euphorbiaceae e Rubiaceae (três espécies cada) e Clusiaceae, Moraceae, Polypodiaceae e Vochysiaceae (duas espécies cada). Na categoria ornamental: Polypodiaceae. Nas demais categorias (30\%) destacam-se: Mimosaceae (quatro espécies), Clusiaceae, Rutaceae e Vochysiaceae (duas espécies cada).
Entre as plantas usadas como remédio $(65,6 \%)$ verificou-se que a maior freqüência destina-se ao tratamento de problemas referentes às afecções do sangue e órgãos hematopoiéticos (17,5\%). Em segundo lugar, para as afecções do sistema circulatório $(14,5 \%)$, seguidas de usos para combater doenças infecciosas e parasitárias (12,6\%), afecções do aparelho geniturinário $(9,5 \%)$. Do aparelho digestivo $(9,5 \%)$ apontaram como auxiliares nos tratamentos de úlceras e gastrites, principalmente. Poucas são indicadas para usos referentes às afecções da pele $(4,6 \%)$ e para o sistema nervoso, sistema osteomuscular e tecido conjuntivo e neoplasias $(3,1 \%)$ (Tab. 5).

A multiplicidade de uso das plantas variou entre um e quatro afecções orgânicas, destacando-se Cariniana rubra (gastrite, hemorróida, inflamação da garganta e inflamação dos ovários); a copaíba (antinflamatório, depurativo do sangue, cicatrizante); o barabatimão (úlcera, inflamação, ferida). A maioria das plantas recebeu, em média, entre dois e três usos terapêuticos pela população de Conceição-Açu.

A parte da planta que obteve o maior percentual de uso foi a folha $(48,3 \%)$, seguido da casca do caule $(23 \%)$ e da raiz $(6,8 \%)$. Entre as formas de preparo utilizadas pela população local a mais expressiva é o chá, com $63 \%$; outras formas também foram relatadas como xarope, infusão, emplastro, compressas, banhos e garrafada.

As plantas usadas como remédio pela população local alcançaram maior importância quando referidas para problemas mais simples e que fazem parte da atenção primária em saúde, por exemplo: gripe, febre, resfriados, gastrite, úlcera, problemas do fígado e estômago, feridas, tonturas, inflamação do útero e ovário.

Das 101 espécies de mata de galeria referidas pelos informantes para um ou mais tipos de usos a maioria é nativa e encontra-se distribuída em todos os estratos da vegetação, com predominância para o arbóreo.

A utilização das espécies ocorre, preferencialmente, em função da proximidade do local de coleta com a residência. $\mathrm{O}$ extrativismo prevalece como forma de obtenção da maioria das espécies, principalmente as folhas para ervas e arbustos, seguido da casca do caule para árvores. As sementes, frutos e raízes são utilizadas em menores proporções.

Os tratamentos de saúde por meio da medicina popular coexistem, paralelamente, aos serviços de saúde fundamentada pelos próprios valores encontrados 
Tabela 4. Plantas da mata de galeria usadas pela comunidade de Conceição-Açu, MT, Brasil. Categorias de uso (Cat. de uso): A = Alimentar; $\mathrm{R}=$ Remédio; Or = Ornamental; Ot = Outros: madeira, artesanato, lenha. Valor de uso das espécies = Vusp; Ref = coletor e número $(\mathrm{MCP}=$ M.C. Pasa); Hábito: HB = Herbácea; SA = Subarbustivo; AB = Arbustivo; AV = Arbóreo; PAL = Palmeira; EPI = Epífita; $\mathrm{TR}=$ Trepadeira. Origem: $\mathrm{N}=$ Nativa; $\mathrm{C}=$ Cultivada; $\mathrm{E}=$ Espontânea.

\begin{tabular}{|c|c|c|c|c|c|c|c|}
\hline Nome popular & Nome científico & Família & at. de uso & Vusp & Hábito & Origem & Ref. HUFMT \\
\hline Algodãozinho & Brosimum gaudichaudii Trécul. & Moraceae & $\mathrm{R}$ & 1,00 & $\mathrm{AB}$ & $\mathrm{N}$ & MCP174 \\
\hline Amescla & Protium heptaphyllum (Aubl.) Marchand. & Burseraceae & $\mathrm{R}$ & 1,00 & AV & $\mathrm{N}$ & MCP106 \\
\hline Angélica, tiborna & Himatanthus obovatus Müll. Arg. & Apocynaceae & $\mathrm{R}$ & 1,00 & $\mathrm{AB}$ & $\mathrm{N}$ & MCP150 \\
\hline Angico & Anadenanthera falcata (Benth.) Spreng. & Mimosaceae & $\mathrm{Ot}$ & 1,50 & AV & $\mathrm{N}$ & MCP153 \\
\hline Angico-branco & Anadenanthera peregrina (L.) Spreng. & Mimosaceae & $\mathrm{Ot}$ & 2,00 & AV & $\mathrm{N}$ & MCP110 \\
\hline Angico-jacaré & Anadenanthera peregrina (L.) Spreng. & Mimosaceae & $\mathrm{Ot}$ & 1,50 & AV & $\mathrm{N}$ & MCP141 \\
\hline Aricá & Physocalymma scaberrim Pohl. & Lythraceae & $\mathrm{Ot}$ & 1,33 & AV & $\mathrm{N}$ & MCP87 \\
\hline Aroeira & Myracroduon urundeuva (Fr. All.) Engl. & Anacardiaceae & $\mathrm{Ot}$ & 2,00 & AV & $\mathrm{N}$ & MCP135 \\
\hline Assa-peixe & Vernonia ferruginea Less. & Asteraceae & $\mathrm{R}$ & 1,66 & HB & $\mathrm{N}$ & MCP130 \\
\hline Ata-do-mato & Annona dioica A. St.-Hil. & Annonaceae & A & 1,00 & AV & $\mathrm{N}$ & MCP89 \\
\hline Babaçu & Orbignya oleifera Burret. & Arecaceae & $\mathrm{A}, \mathrm{Ot}$ & 2,50 & PAL & $\mathrm{N}$ & MCP98 \\
\hline Bacuri & Rheedia brasiliensis Camb. & Clusiaceae & $\mathrm{R}, \mathrm{Ot}$ & 1,50 & AV & $\mathrm{N}$ & MCP123 \\
\hline Banana & Musa paradisiaca Kuntze & Musaceae & A & 1,50 & $\mathrm{AB}$ & $\mathrm{C}$ & MCP184 \\
\hline Barbatimão & Stryphnodendron adstringens (Mart.) Coville & Mimosaceae & $\mathrm{R}$ & 1,50 & AV & $\mathrm{N}$ & MCP139 \\
\hline Bocaiúva & Acrocomia aculeata (Jacq.) Lodd. & Arecaceae & A & 1,00 & PAL & $\mathrm{N}$ & MCP132 \\
\hline Cabaça-coite & Crescentia cujete $\mathrm{L}$. & Bignoniaceae & $\mathrm{R}$ & 1,00 & $\mathrm{AV}$ & $\mathrm{N}$ & MCP129 \\
\hline Cabriteiro & Rhamnidium elaeocarpum Reiss. & Rhamnaceae & $\mathrm{Ot}$ & 1,00 & $\mathrm{AB}$ & $\mathrm{N}$ & MCP175 \\
\hline Café & Coffea arabica $\mathrm{L}$. & Rubiaceae & A & 1,33 & $\mathrm{AB}$ & $\mathrm{C}$ & MCP163 \\
\hline Cambará-do-mato & Vochysia haenkeana Mart. & Vochysiaceae & $\mathrm{Ot}$ & 1,33 & AV & $\mathrm{N}$ & MCP136 \\
\hline Cana-de-macaco & Philodendron sp. & Araceae & $\mathrm{R}$ & 1,00 & HB & $\mathrm{C}$ & MCP142 \\
\hline Cancerosa & Synadenium grantii Hook. F. & Euphorbiaceae & $\mathrm{R}$ & 1,00 & $\mathrm{AB}$ & $\mathrm{C}$ & MCP108 \\
\hline Canjiquinha & Byrsonima intermedia A. Juss. & Malpighiaceae & $\mathrm{Ot}$ & 1,00 & AV & $\mathrm{N}$ & MCP173 \\
\hline Cansanção & Jatropha urens L. & Euphorbiaceae & $\mathrm{R}$ & 1,00 & SA & $\mathrm{N}$ & MCP92 \\
\hline Carobinha-branca & Jacaranda decurrens Cham. & Bignoniaceae & $\mathrm{R}$ & 1,00 & $\mathrm{AB}$ & $\mathrm{N}$ & MCP151 \\
\hline Carvão-branco & Callisthene fasciculata (Spr.) Mart. & Vochysiaceae & $\mathrm{Ot}$ & 1,33 & AV & $\mathrm{N}$ & MCP178 \\
\hline Carvão-vermelho & Diptychandra aurantiaca Tul. & Caesalpiniaceae & Ot & 2,00 & AV & $\mathrm{N}$ & MCP134 \\
\hline Catipé & Licania sp. & Chrysobalanaceae & Ot & 1,00 & AV & $\mathrm{N}$ & MCP138 \\
\hline Catuaba & Anemopaegma arvense (Vell.) S. & Bignoniaceae & $\mathrm{R}$ & 1,00 & SA & $\mathrm{N}$ & MCP144 \\
\hline Chico-magro & Guazuma ulmifolia Lam. & Sterculiaceae & $\mathrm{R}$ & 1,00 & AV & $\mathrm{N}$ & MCP124 \\
\hline Cipó-caboclo & Doliocarpus sp. & Dilleniaceae & $\mathrm{R}$ & 1,00 & TR & $\mathrm{N}$ & MCP148 \\
\hline Copaíba & Copaifera langsdorffii Desf. & Caesalpiniaceae & $\mathrm{R}$ & 2,50 & $\mathrm{AV}$ & $\mathrm{N}$ & MCP88 \\
\hline Coração-de-negro & Crisophyllum sp. & Sapotaceae & $\mathrm{Ot}$ & 1,00 & AV & $\mathrm{N}$ & MCP170 \\
\hline Coroa-de-frade & Mouriria elliptica Mart. & Melastomataceae & $\mathrm{R}$ & 1,00 & AV & $\mathrm{N}$ & MCP133 \\
\hline Cumbarú & Dipteryx alata Vog. & Fabaceae & $\mathrm{Ot}$ & 1,75 & AV & $\mathrm{N}$ & MCP152 \\
\hline Dorme-dorme & Mimosa adenocarpa Benth. & Mimosaceae & $\mathrm{R}$ & 1,50 & AV & $\mathrm{N}$ & MCP162 \\
\hline Douradinha & Palicourea xanthophylla Muell. Arg. & Rubiaceae & $\mathrm{R}$ & 1,00 & AS & $\mathrm{N}$ & MCP180 \\
\hline Embaúba & Cecropia pachystachya Trècul. & Moraceae & $\mathrm{R}$ & 1,00 & AV & $\mathrm{E}$ & MCP179 \\
\hline Erva-de-bicho & Polygonum acre H.B.K. & Polygonaceae & $\mathrm{R}$ & 1,00 & $\mathrm{HB}$ & $\mathrm{N}$ & MCP118 \\
\hline Espicha-couro & Xylopia aromática (Lam.) Mart. & Annonaceae & $\mathrm{Ot}$ & 1,00 & $\mathrm{AV}$ & $\mathrm{C}$ & MCP144 \\
\hline Fruta-do-conde & Annona reticulata $\mathrm{L}$. & Annonaceae & $\mathrm{A}, \mathrm{R}$ & 1,00 & $\mathrm{AV}$ & $\mathrm{C}$ & MCP121 \\
\hline Gervão & Stachytarpheta angustifolia Lopez-Palacios & Verbenaceae & $\mathrm{R}$ & 1,00 & HB & $\mathrm{E}$ & MCP155 \\
\hline Gravatá & Bromelia balansae Mez. & Bromeliaceae & $\mathrm{R}$ & 1,00 & HB & $\mathrm{N}$ & MCP99 \\
\hline Guanandi & Callophyllum brasiliensis Camb. & Clusiaceae & $\mathrm{R}$ & 1,00 & AV & $\mathrm{N}$ & MCP143 \\
\hline Guaraná & Paullinia cupana H.B.K. & Sapindaceae & $\mathrm{A}, \mathrm{R}$ & 1,50 & TR & $\mathrm{C}$ & MCP181 \\
\hline Guatambu & Aspidosperma polyneuron Muell. Arg. & Apocynaceae & Ot & 2,50 & AV & $\mathrm{N}$ & MCP127 \\
\hline Hortelã-do-brejo & Hyptis goyazensis A. St.-Hil. ex. Benth & Lamiaceae & $\mathrm{R}$ & 1,66 & $\mathrm{HB}$ & $\mathrm{C}$ & MCP95 \\
\hline Ingá & Inga $\mathrm{sp}$ & Mimosaceae & $\mathrm{R}$ & 1,50 & AV & $\mathrm{N}$ & MCP125 \\
\hline Ipê-amarelo & Tabebuia ochracea Standl. & Bignoniaceae & $\mathrm{R}$ & 2,00 & AV & $\mathrm{N}$ & MCP147 \\
\hline Ipê-roxo & Tabebuia heptaphylla (Vell.) Toledo & Bignoniaceae & $\mathrm{R}$ & 2,00 & AV & $\mathrm{N}$ & MCP122 \\
\hline Jaborandi-da-mata & Ottonia corcovadensis Miq. & Piperaceae & $\mathrm{R}$ & 1,00 & $\mathrm{AB}$ & $\mathrm{N}$ & MCP166 \\
\hline Jatobá & Hymenaea stignocarpa Mart. & Caesalpiniaceae & $\mathrm{R}$ & 2,33 & AV & $\mathrm{N}$ & MCP126 \\
\hline Jequitibá & Cariniana rubra Gardner. ex. Miers & Lecythidaceae & $\mathrm{R}$ & 2,00 & $\mathrm{AV}$ & $\mathrm{N}$ & MCP105 \\
\hline Jurubeba & Solanum aff. lycocarpum A. St.-Hil. & Solanaceae & $\mathrm{R}$ & 1,00 & $\mathrm{AB}$ & $\mathrm{N}$ & MCP101 \\
\hline Limão & Citrus limonum Osbeck. & Rutaceae & $\mathrm{R}$ & 1,00 & $\mathrm{AB}$ & $\mathrm{C}$ & MCP165 \\
\hline
\end{tabular}


Tabela 4 (continuação)

\begin{tabular}{|c|c|c|c|c|c|c|c|}
\hline Nome popular & Nome científico & Família & Cat. de uso & Vusp & Hábito & Origem & Ref. HUFMT \\
\hline Língua-de-vaca & Orthopappus angustifolius (Sw.) Gleason. & Asteraceae & $\mathrm{R}$ & 1,00 & $\mathrm{HB}$ & $\mathrm{C}$ & MCP93 \\
\hline Lixeira & Curatella americana $\mathrm{L}$. & Dilleniaceae & $\mathrm{R}$ & 1,66 & AV & $\mathrm{N}$ & MCP160 \\
\hline Lixinha & Davilla nitida (Vahl.) Kubitz & Dilleniaceae & $\mathrm{R}$ & 1,00 & $\mathrm{AB}$ & $\mathrm{N}$ & MCP116 \\
\hline Louro-branco & Cordia glabrata Mart. & Boraginaceae & A & 1,00 & AV & $\mathrm{N}$ & MCP111 \\
\hline Mamica-de-porca & Zanthoxylum rhoifolium Lam. & Rutaceae & $\mathrm{Ot}$ & 1,00 & $\mathrm{AV}$ & $\mathrm{N}$ & MCP113 \\
\hline Mangaba & Hancornia speciosa Gomez & Apocynaceae & $\mathrm{R}$ & 1,50 & $\mathrm{AV}$ & $\mathrm{N}$ & MCP146 \\
\hline Mangava-brava & Lafoensia pacari A. St.-Hil. & Lythraceae & $\mathrm{R}$ & 2,00 & AV & $\mathrm{N}$ & MCP112 \\
\hline Maracujá-do-mato & Passiflora sp. & Passifloraceae & $\mathrm{R}$ & 1,00 & $\mathrm{TR}$ & $\mathrm{N}$ & MCP159 \\
\hline Maria-pobre & Dilodendron bipinnatum Radlk. & Sapindaceae & $\mathrm{R}$ & 1,33 & AV & $\mathrm{N}$ & MCP177 \\
\hline Marmelada-bola & Alibertia edulis (L.R.) A. Rich ex DC. & Rubiaceae & $\mathrm{R}$ & 1,00 & $\mathrm{AB}$ & $\mathrm{N}$ & MCP102 \\
\hline Marmelada-espinho & Alibertia verrucosa S. Moore & Rubiaceae & A & 1,00 & $\mathrm{AB}$ & $\mathrm{N}$ & MCP182 \\
\hline Negramina & Siparuna guianensis Aubl. & Monimiaceae & $\mathrm{R}$ & 1,50 & AV & $\mathrm{N}$ & MCP167 \\
\hline Olho-de-pomba & Coussarea hydrangeaefolia Benth. \& Hook. & Rubiaceae & $\mathrm{R}$ & 1,00 & AV & $\mathrm{N}$ & MCP176 \\
\hline Paratudo & Tabebuia aurea (M.) Benth \& Hook. & Bignoniaceae & $\mathrm{R}$ & 2,00 & $\mathrm{AB}$ & $\mathrm{N}$ & MCP96 \\
\hline Pau-d'anta & Cybistax antisyphyllitica Mart. & Bignoniaceae & $\mathrm{R}$ & 1,00 & AV & $\mathrm{N}$ & MCP145 \\
\hline Pau-doce & Vochysia rufa Mart. & Vochysiaceae & $\mathrm{R}$ & 1,00 & AV & $\mathrm{N}$ & MCP164 \\
\hline Pau-santo & Kielmeyera coriacea Mart. & Clusiaceae & $\mathrm{Ot}$ & 1,50 & AV & $\mathrm{N}$ & MCP149 \\
\hline Pau-terra & Qualea grandiflora Mart. & Vochysiaceae & $\mathrm{R}$ & 1,50 & AV & $\mathrm{N}$ & MCP90 \\
\hline Pau-terra-macho & Qualea multiflora Mart. & Vochysiaceae & $\mathrm{Ot}$ & 1,00 & AV & $\mathrm{N}$ & MCP140 \\
\hline Pequi & Caryocar brasiliense Camb. & Caryocaraceae & $\mathrm{A}$ & 1,75 & $\mathrm{AV}$ & $\mathrm{N}$ & MCP115 \\
\hline Periquiteiro & Trema micrantha (L.) Blume & Ulmaceae & $\mathrm{Ot}$ & 1,00 & AV & $\mathrm{E}$ & MCP154 \\
\hline Peroba & Aspidosperma australe Muell. Arg. & Apocynaceae & $\mathrm{R}$ & 1,00 & V & $\mathrm{N}$ & MCP91 \\
\hline Pitomba & Talisia esculenta (A. St.-Hil.) Radlk. & Sapindaceae & A & 1,33 & AV & $\mathrm{N}$ & MCP168 \\
\hline Quina-genciana & Acosmium subelegans (Mohl.) Yak. & Fabaceae & $\mathrm{R}$ & 2,00 & $\mathrm{AV}$ & $\mathrm{N}$ & MCP157 \\
\hline Rabo-de-caxinguelê & Phlebodium decumanum (Willd.) J. Sm. & Polypodiaceae & $\mathrm{R}$ & 1,00 & EPI & $\mathrm{N}$ & MCP183 \\
\hline Salsa-paredão & Caladium sp. & Araceae & $\mathrm{R}$ & 1,00 & $\mathrm{HB}$ & $\mathrm{N}$ & MCP156 \\
\hline Samambaia-mato & Polypodium sp. & Polypodiaceae & Or & 1,00 & EPI & $\mathrm{N}$ & MCP171 \\
\hline Sangra-d'água & Croton salutaris Muell. Arg. & Euphorbiaceae & $\mathrm{R}$ & 2,50 & $\mathrm{AV}$ & $\mathrm{N}$ & MCP100 \\
\hline Serigüela & Spondias lutea $\mathrm{L}$. & Anacardiaceae & A & 1,00 & AV & $\mathrm{C}$ & MCP120 \\
\hline Sucupira-branca & Pterodon pubescens Benth. & Fabaceae & $\mathrm{R}$ & 1,50 & $\mathrm{AV}$ & $\mathrm{N}$ & MCP119 \\
\hline Sucupira-preta & Bowdichia virgiloides H.B.K. & Fabaceae & $\mathrm{R}$ & 1,50 & AV & $\mathrm{N}$ & MCP107 \\
\hline Taioba & Colocasia esculenta $\mathrm{L}$ & Araceae & $\mathrm{R}$ & 1,00 & $\mathrm{HB}$ & $\mathrm{C}$ & MCP161 \\
\hline Tamarindo & Tamarindus indica $\mathrm{L}$. & Caesalpiniaceae & $\mathrm{R}$ & 2,00 & AV & $\mathrm{C}$ & MCP172 \\
\hline Tarumã & Vitex cymosa Bert. & Verbenaceae & $\mathrm{R}$ & 1,00 & AV & $\mathrm{N}$ & MCP117 \\
\hline Tarumarana & Buchenavia tomentosa Eichl. & Combretaceae & $\mathrm{R}$ & 1,00 & AV & $\mathrm{N}$ & MCP104 \\
\hline Timbó & Magonia pubescens A. St.-Hil. & Sapindaceae & $\mathrm{R}$ & 1,00 & AV & $\mathrm{N}$ & MCP128 \\
\hline Tripa-de-galinha & Bauhinia glabra Jacq. & Caesalpiniaceae & $\mathrm{R}$ & 1,00 & $\mathrm{TR}$ & $\mathrm{N}$ & MCP97 \\
\hline Tucum & Bactris glaucescens Drude & Arecaceae & A & 2,00 & PAL & $\mathrm{N}$ & MCP131 \\
\hline Unha-de-boi & Bauhinia sp. & Caesalpiniaceae & $\mathrm{R}$ & 1,75 & $\mathrm{AB}$ & $\mathrm{N}$ & MCP158 \\
\hline Urucum & Bixa orellana $\mathrm{L}$. & Bixaceae & $\mathrm{A}$ & 1,66 & $\mathrm{AB}$ & $\mathrm{C}$ & MCP114 \\
\hline Uva-brava & Cissus erosa Rich. & Vitaceae & $\mathrm{R}$ & 1,00 & $\mathrm{TR}$ & $\mathrm{N}$ & MCP169 \\
\hline Vinhático & Plathymenia reticulata Benth. & Mimosaceae & $\mathrm{Ot}$ & 1,00 & AV & $\mathrm{N}$ & MCP103 \\
\hline Ximbuva & Enterolobium contortisiliquum (Vell.) M. & Mimosaceae & $\mathrm{Ot}$ & 1,00 & AV & $\mathrm{N}$ & MCP109 \\
\hline
\end{tabular}

pela população local e também pela transmissão da herança cultural. Nesse sentido, diante da realidade em que vive a comunidade local, o que prevalece é o empírico, de modo que a maioria das plantas usadas como medicinais pertence à flora mato-grossense e o investimento em estudos farmacológicos proporcionaria resultados expressivos, contribuindo para o uso das espécies referidas com maior precisão.

Nos quintais e roças a preferência de uso das plantas é quase equivalente, como alimento $(48,1 \%)$ e medicinal (44,5\%). Nas matas de galeria e adjacências a categoria medicinal é predominante $(65,6 \%)$ e a de alimento menos expressiva, fato que demonstra a diversidade vegetal do ambiente e a conseqüente diversidade cultural para uso das plantas. Quanto ao valor de uso das espécies nativas destacaram-se: a copaíba, o carvão-vermelho, o jequitibá, o paratudo, a quina-genciana, o guatambu, o ipê-amarelo, o ipê-roxo, a sangra-d'água, a aroeira, o babaçu, o jatobá, a mangava-brava, o tucum, entre outros. Na categoria 
Tabela 5. Espécies botânicas usadas como remédio nas diferentes afecções orgânicas referidas pela comunidade de Conceição-Açu, Cuiabá, MT, Brasil, obtidas na mata de galeria.

\begin{tabular}{|c|c|c|}
\hline Categoras & Espécies & Usos \\
\hline Doenças do sistema nervoso & maracujá-do-mato, dorme-dorme & Ansiedade, calmante, sistema nervoso \\
\hline Doenças do aparelho digestivo & $\begin{array}{l}\text { jequitibá, mangava-brava, marmelada- } \\
\text { bola, olho-de-pomba, jurubeba, gervão, } \\
\text { pau-terra }\end{array}$ & Gastrite, úlcera, dor de estômago \\
\hline Doenças do aparelho respiratório & $\begin{array}{l}\text { assa-peixe, cabaça-coité, amescla, } \\
\text { hortelã-do-brejo, ingá, embaúba, } \\
\text { sucupira-branca }\end{array}$ & $\begin{array}{l}\text { Gripe, febre, tosse, bronquite, sinusite, } \\
\text { constipação nasal }\end{array}$ \\
\hline Doenças do aparelho circulatório & $\begin{array}{l}\text { salsa-paredão, urucum, guanandi, } \\
\text { copaíba, coroa-de-frade, negramina, } \\
\text { taioba, tripa-de-galinha, sucupira-preta }\end{array}$ & $\begin{array}{l}\text { Pressão alta, varize, prurido, hemorróida, } \\
\text { malina, ácido úrico, depurativo do sangue }\end{array}$ \\
\hline Doenças do aparelho geniturinário & $\begin{array}{l}\text { lixeira, carobinha-branca, tamarindo, } \\
\text { embaúba, douradinha, rabo-de-caxin- } \\
\text { guelê }\end{array}$ & $\begin{array}{l}\text { Doença venérea, pedra nos rins, bexiga, } \\
\text { cistite, rins, inflamação no canal da urina }\end{array}$ \\
\hline Afecções sistema tegumentar & chico-magro, cipó-caboclo, tarumarana & Alergia, irrritação da pele, feridas \\
\hline Doenças do olho e anexos & bacuri & Dor dói (dor no olho) \\
\hline Doenças infecciosas e parasitárias & $\begin{array}{l}\text { jaborandi-da-mata, douradinha, lixinha, } \\
\text { salsa-paredão, cabaçá-coité, tiborna, } \\
\text { sucupira-preta, maria-pobre }\end{array}$ & $\begin{array}{l}\text { Limpa intestino, tuberculose, dor de } \\
\text { barriga, depurativo, verme, prurido anal }\end{array}$ \\
\hline Doenças do sistema osteomuscular e conjuntivo & pau-d'anta, pau-terra-macho, aroeira & Dor nas cadeiras, arca caída \\
\hline Neoplasias & ipê-roxo, ipê-amarelo & Início de câncer, câncer \\
\hline Doenças do sangue e órgãos hematopoiéticos & $\begin{array}{l}\text { fruta-do-conde, taioba, língua-de-vaca, } \\
\text { carobinha-branca, paratudo, sangra- } \\
\text { d'água, guanandi, jatobá, jequitibá, } \\
\text { copaíba, sucupira-preta }\end{array}$ & $\begin{array}{l}\text { Anemia, depurativo do sangue, fraqueza, } \\
\text { reumatismo }\end{array}$ \\
\hline
\end{tabular}

de uso medicinal destacaram-se as famílias Mimosaceae, Bignoniaceae, Caesalpiniaceae, Fabaceae e Sapindaceae. A comunidade de ConceiçãoAçu concebe os recursos vegetais dentro de um significado muito amplo de utilidade, apresentando uma dependência dos recursos localmente disponíveis, especialmente de plantas medicinais, na mata de galeria e alimentar, nas roças e quintais. Os usos descritos para a mata de galeria revelam expressivo aproveitamento em relação às funções de coleta, demonstrando preocupação com a manutenção das potencialidades vegetais contribuindo, dessa forma, para a conservação da biodiversidade nos ecossistemas naturais.

\section{Referências bibliográficas}

Almeida, C.F.C.B.R. \& Albuquerque, U.P. 2002. Uso e conservação de plantas e animais medicinais no Estado de Pernambuco (Nordeste do Brasil): um estudo de caso. Interciência 27(6): 276-285.

Albuquerque, U.P. \& Andrade, L.H.C. 2002. Uso de recursos vegetais da caatinga: o caso do Agreste do Estado de Pernambuco(Nordeste do Brasil). Interciência 27(7): 336-346.
Amorozo, M.C.M. 2000. Management and conservation of Manihot esculenta Crantz. germ. plasm by tradicional farmers in Santo Antonio do Leverger, Mato Grosso State, Brazil. Etnoecologica 4(6): 69-83.

Amorozo, M.C.M. \& Gely, A. 1988 Uso de plantas medicinais por caboclos do Baixo Amazonas. Barcarena, PA, Brasil. Boletim do Museu Paraense Emílio Goeldi, sér. Bot. 4: 47-131.

Barrera, A. 1979. La Etnobotânica. Pp. 19-25. In: A. Barrera. La Etnobotânica: três puntos de vista e uma perspectiva. Xalapa, México, Instituto de Investigacion sobre Recursos Bióticos.

Begossi, A. 2001. Espaços e recursos naturais de uso comum. Pp. 205-231. In: Resiliência e populações neotradicionais: os caiçaras (Mata Atlântica) e os caboclos (Amazônia, Brasil). São Paulo. NUPAUB, USP.

Berg, M.E. van den. 1980. Contribuição à flora medicinal do estado do Mato Grosso. In: Simpósio de Plantas Medicinais do Brasil. Suplemento Ciência e Cultura 33: $163-17$.

Caballero, J. 1979. La Etnobotânica. Pp. 27-30. In: A. Barrera (ed.). La Etnobotânica: tres puntos de vista y uma perspectiva. Xalapa, INIREB.

Guarim-Neto, G. 1984. Plantas utilizadas na medicina popular cuiabana - um estudo preliminar. Revista da Universidade Federal de Mato Grosso 4(1): 45-50. 
Guarim-Neto, G. 1996. Plantas Medicinais do Estado do Mato Grosso, Brasília, ABEAS, UFMT.

Minayo, M.C.S. 1994. O desafio do conhecimento. Pesquisa qualitativa em saúde. São Paulo, HUCITEC; ABRASCO.

Organização Mundial de Saúde. 2000. Classificação Estatística Internacional de Doenças e Problemas Relacionados à Saúde. São Paulo, Editora da Universidade de São Paulo.

PCBAP. 1997. Programa Nacional do Meio Ambiente: Diagnóstico dos Meios Físico e Biótico 2(1): 32-38.
Phillips, O. \& Gentry, A.H. 1993. The useful plants of Tambopata, Peru. I. Statistical hypotheses with a new quantitative technique. Economic Botany 47(1): 33-43.

Phillips, O.L. 1996. Some quantitative methods for analyzing ethnobotanical knowledge. In: M.N. Alexiades (ed.). Selected Guidelines for Ethnobotanical Research: a field manual. New York, New York Botanical Garden.

RADAMBRASIL. 1982. Levantamento de Recursos Naturais. Folha SD 21, Cuiabá. v.26. Brasília, MME/ SG. Strabler, A.N. \& Strabler, A.H. 1989. Geografia Física. Barcelona, Ediciones Omega S.A. Platô, 2. 